\title{
Multimodal integration of visual place cells and grid cells for navigation tasks of a real robot
}

\author{
Adrien Jauffret, Nicolas Cuperlier, Philippe Gaussier and Philippe Tarroux \\ ETIS laboratory, UMR 8051 \\ F-95000 - Cergy Cedex, France. \\ LIMSI laboratory, CNRS(UPR3251) \\ 91403 Orsay Cedex, France.
}

\begin{abstract}
In the present study, we propose a model of multimodal place cells merging visual and proprioceptive primitives. First we will briefly present our previous sensory-motor architecture, highlighting limitations of a visual-only based system. Then we will introduce a new model of proprioceptive localization, giving rise to the so-called grid cells, wich are congruent with neurobiological studies made on rodent.

Finally we will show how a simple conditionning rule between both modalities can outperform visual-only driven models by producing robust multimodal place cells. Experiments show that this model enhances robot localization and also allows to solve some benchmark problems for real life robotics applications.
\end{abstract}

Keywords: Grid cells; Bio-inspired robotics; Multimodal integration; Sensory-motor navigation \& mapping; Neural networks.

\section{Introduction}

Ethological studies of animal navigation show that a wide variety of sensory modalities can be used by animals to navigate and self localize in an unknown and complex environment. Since the startling discovery by OKeefe and Dostrovsky [1] of the spatial correlates of neural activity in the hippocampal system (HS) of rodents, some work has been done to investigate the neural bases of animals spatial learning (see [2] for a short review). Originally found in HS, place cells are pyramidal neurons exhibiting high firing rates at a particular location in the environment (place field). Cells with similar properties but with larger place field have also been found in the Enthorinal Cortex (EC). As firing of place cells persists in the dark it has also been suggested that other senses (proprioception, touch, smell) might contribute as well [3-8].

Later in 2005, Hafting and Moser discorvered grid cells in the dorso-lateral band of the medial EC (dMEC) [9]. These cells present spatial firing fields forming regular triangle-pattern (grid) that tiled the environment. They could be the basis of a cognitive map of Euclidean space. Each grid is defined by 3 parameters: frequency (distance between two vertex), phase (spatial shift) and orientation. Grid cell activity does not require visual input, since it remains unchanged in 
absence of any visual cue (dark) even if the bumps of activity tend to spread due to accumulation of errors by the integration process [9].

Three main classes of models have been proposed: recurrent network models based on continuous attractor dynamics $[10,11]$, independent-neuron models based on oscillatory interference [12] and models using a residue number system [13].

Following our previous work [14] based on a residue number model, we first present a robotic implementation of this model able to exhibit grid cells like firing pattern. Results underline the key role played by visual inputs. We show that without visual recalibration, grid cells firing seems scrambled, according to biological results [9]. Next we present a model, based on a pavlovian conditioning rule, that merges signals coming from visual cells and grid cells into multimodal place cells. Experiments on a real robot show how grid cells information can be enough to self-localize in the dark on short distances. We also show how grid cells activity help to greatly reduce visual ambiguity, giving robust multimodal place fields. Finally, we will briefly discuss how this model behaves when the robot is kidnapped and shifted to another location.

\section{Modeling Place Cells from visual information}

In previous works, we developped a model of the hippocampus in order to obtain visual place cells (VPCs) [15] that allowed controlling mobile robots for visual navigation tasks $[16,17]$. The embedded pan-tilt camera allows the capture of several images (actually 15) corresponding to a 360 degrees panorama. A gradient image convolved with a DoG (difference of gaussian) filter allows to highlight a set of salient points in the scene (curvature points at a low resolution). A log polar transform of a small circular image centered on each focus point (local view of $16^{*} 16$ pixels) is computed in order to improve the pattern recognition against small rotations and scale variations.

Then, a neural network learns place cells that code information about a constellation of landmarks in the scene (5 landmarks per images) (figure 1). Activities of the different place cells depend on the recognition level of landmarks. Robustness comes from the large number of local views extracted ( 75 per panorama) and the only use of a competition between place cells (see [18] for detailed parameters). In our model, local views correspond to the "what" information coded in the perirhinal cortex or in other areas of the ventral visual pathway of the rat temporal cortex [19]. The absolute position of these local views (the "where" information) is provided by the parietal cortex through the parahippocampal region. The merging of "what" and "where" information may be performed in the superficial layer of the enthorinal cortex or in the postrhinal cortex [20,21].

A neural network learns to associate a particular PCs with an action (a direction to follow in our case). This sensory-motor architecture is named PerAc [22] and allows the robot to learn simple but robust behaviors.

Even if our architecture has been succesfully tested in small sized environments 


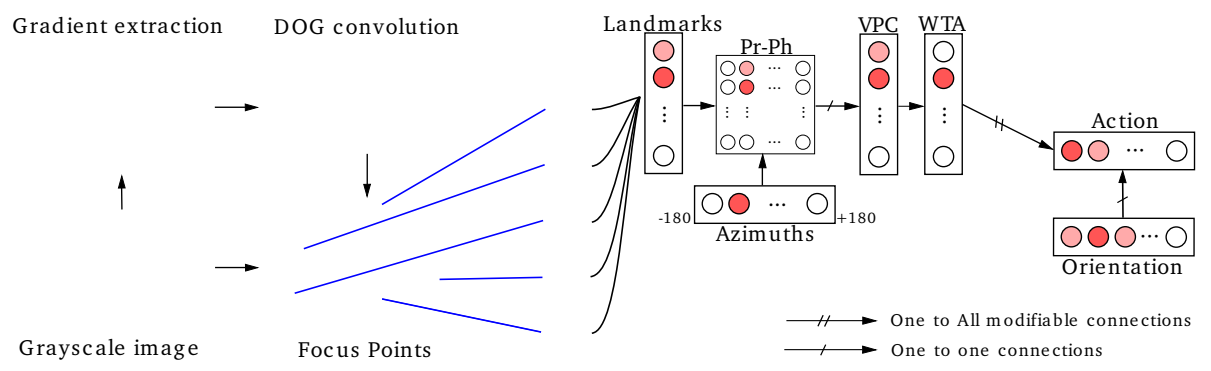

Fig. 1. Sensorimotor model relying on vision. The gradient image is convolved with a difference of gaussian filter. Local maxima of the resulting image correspond to points of interest on which the system focuses on to extract local views. A Place Cell (PC) learns to recognize a specific landmarksazitmuths constellation. An action is associated with this PC. This association is learned by a least mean square algorithm (LMS), after what the system is able to move in the learned direction when the associated PC wins.

(typically one room), a visual-only based mechanism shows limitations when trying to scale to larger and more complex ones (multi-room, outdoors). First the large number of PCs needed to cover this kind of environment introduces a computational problem that highly decreases the robustness of the localization. Then a lot of mistakes are due to the ambiguous nature of the visual modality. Indeed the activity of a PC can highly responds for different locations with identical visual panorama (corridor for instance). We propose to overcome these issues by adding a bio-inspired localization mechanism based on proprioception, following our hypothesis of the way grid cells work.

\section{Modeling Grid Cells from extra hippocampal path integration}

\subsection{A plausible model of grid cells}

Here we present a model for generating grid cells from path integration. Our simplest model of grid cells (GCs) is based on various modulo's operator applied on path integration [14] (figure 2). The path integrator is supposed to be stored outside the hippocampal system. The activity $D_{i}$ of a neuron belonging the path integration field (associated with direction $\theta_{i}$ ) is discretized over a new field of neurons $E_{j}^{i}=\operatorname{round}\left(\frac{D_{i} . N_{E}}{D_{\max }}\right)$ where $D_{\max }$ is the maximum value of the distance that can be computed by the neural field, $N_{E}$ is the number of neurons on each field used to discretized each analog activity on the path integration field. Then a modulo operator is used to compress the field $E_{j}^{i}$ by projection.

$$
M_{k}^{n}= \begin{cases}1 & \text { if } k=\operatorname{ArgMax}\left(E_{j}\right) \bmod \lambda^{n} \\ 0 & \text { otherwise }\end{cases}
$$

with $\lambda^{n}$ the value of the modulo used to build grid $n$.

A recalibration mechanism, using a Widrow and Hoff learning rule [24, 26], associate each new VPC with the current path integration field activity (one-shot 
learning), so that it can be recalibrated when that VPC is later well recognized, like $[27,10,28-30]$ (actually when the winning cell activity reach an absolute threshold of 0.9 and a difference relative to VPCs mean activity of 0.4 ).

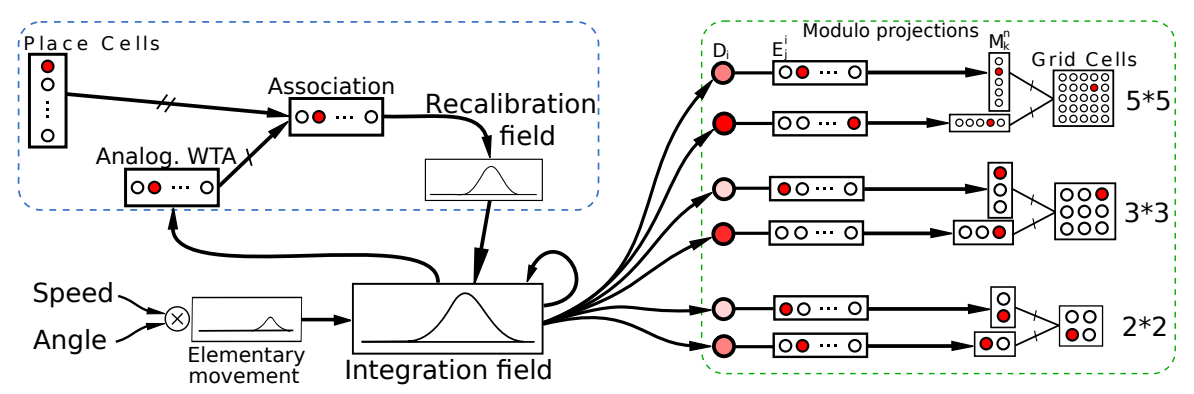

Fig. 2. Linear speed and absolute orientation are used to characterize movement unit and so generate global path integration on a neural field. A recalibration mechanism associate a VPC with the argmax of the neural field (distance and orientation are stored). It allows the system to limit cumulative error on this field when it later well recognizes the corresponding VPC. Path integration field is then used to build grid cell activity without any cartesian map. Activities of randomly chosen pairs of neurons $D_{i}$ in that field are discretized on other fields $E_{i}^{j}$. Those fields are compressed by simple modulo projections $M_{k}^{n}$. The conjonction of 2 codes of 2 projections is sufficient to obtain grid cells.

\subsection{Recreating grid cells activity on 3 experiments with a real robot}

Typical experiments made on rodent consist in recording the activity of grid cells in dMEC while the rat (around $20 \mathrm{~cm}$ large) freely moves in a circular enclosure ( $2 \mathrm{~m}$ of diameter) during 30 minutes. Our experiments run in almost similar conditions since a real robot (around $40 \mathrm{~cm}$ large) randomly moves in an hexagonal park ( $4 \mathrm{~m}$ of diameter) during the same period of time. Position and simulated grid cells are simultaneously recorded. Ultrasound sensors are used by the robot to avoid obstacles and stay inside the hexagonal playground.

In a first experiment, no recalibration is allowed. We note a fast drift of grid cells activity induced by cumulative errors on path integration. All cells present a blurred activity without grid-like pattern because of a 30 minutes errors cumulation (figure 3 ).

In a second experiment, a VPC is learned at the center of the hexagon and visual calibration is allowed. We obtain coherent grid-like pattern but error remains too high to correctly visualize small grids. Error's amplitude is directly linked with the recalibration area (figure 3 ).

To avoid this problem, we made a third experiment with a touch-like reset cue at the corner of 2 walls. The origin of the path integration is set in this corner and a visual attraction field is learned around it by a few sensory-motor associations. It allows the robot to autonomously converges into the reset area by visual recognition (homing behavior). The reset cue is a red paper sticked on the floor and a color detector is used by the robot to perceive this goal. A simple 
counter triggers a periodic drive allowing an homing behavior every minute. The drive is reset each time the goal is reached, allowing the robot to switch back to a random exploration strategy. This solution avoids errors accumulation over more than one minute and so gives us the precision needed to display small grid. But it introduces a static error directly linked with the size of the reset field. Results show well-defined pattern for the different modulo factors. Those grid activities are congruent with neurobiological records in rodents EC [9]. Grid shared the same spacing for the same modulo and the same orientation for the same discretization factor. Nevertheless, each cell produces a grid of different phase.
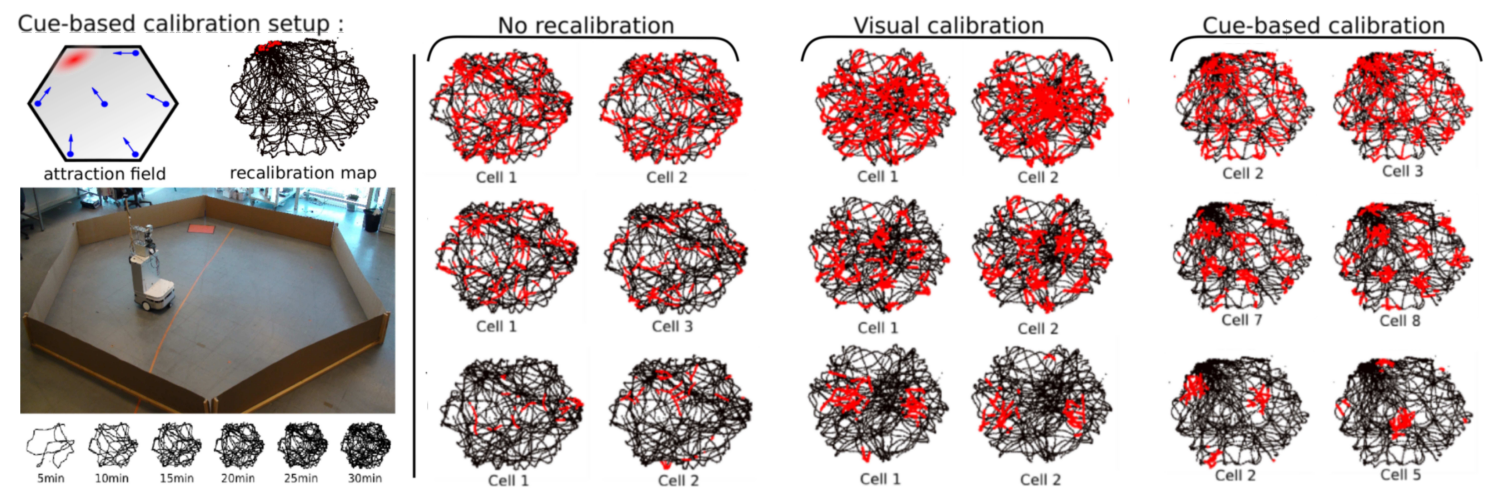

Fig. 3. Experiments made with a real robot (around $40 \mathrm{~cm}$ large) randomly moving in an hexagonal enclosure ( $4 \mathrm{~m}$ of diameter) during 30 minutes (trajectory in black, neural activity in red). Left : Experimental setup: for the cue-based calibration experiment, path integration is set in one corner of the park and an attraction field is learned around it allowing the robot to autonomously go recalibrate itself every minute. Right : Results of the third experiment show coherent regular hexagonal pattern of different phases and modulos. Those patterns are quite similar to thus obtained with rodents.

\section{Building robust multimodal place cells from visual and grid cells}

\subsection{A pavlovian model of merging}

In this section, we propose a simple merging mechanism which can take advantage of allothetic and idiothetic information. This mechanism pops up the synergy between both modalities.

There are many ways of merging different information sources, and it is known as a difficult problem, especially when the nature of the sources are highly different. Sensory modalities must be recoded into a common format before they can be combined. The task is described as the recoding problem by Pouget and Deneve [23].

Our model is based on the learning of associations between VPCs and the whole GCs pattern. The system associates a particular GCs state (conditional stimulus) 
with the current winning VPC (inconditional stimulus) by a classical conditionning rule $[24,26]$, as for the recalibration mechanism (one-shot learning), like [27]. We test it with a normalized least mean square algorithm (NLMS) [25] trying to predict the visual state from GCs activities. A simple weighted sum allows the merging of VPCs and odometric place cells from GCs.

The activity of a multimodal place cell $M P C_{k}$ is given by a simple linear combination of $V P C$ and PredVPC activities:

$$
M P C_{k}=\alpha \cdot V P C_{k}+(1-\alpha) \cdot P r e d V P C_{k}
$$

with $V P C_{k}$ the activity of the corresponding visual place cell, $\operatorname{Pred} V P C_{k}$ the activity of the corresponding place cell predicted by the grids (NLMS output), $\alpha \epsilon[0 ; 1]$ a ponderation factor ( 0.5 in our case) and $k \epsilon[0 ; \mathrm{K}]$ the indice of the cell.

The activity of a place cell predicted by grid cells is given by:

$$
\text { PredVPC } C_{k}=\sum_{k=0}^{K-1} w_{k} \cdot G_{k}
$$

where $w_{k}$ is the weight of the synapse coming from the corresponding grid cell $G_{k}$.

\subsection{Results obtained during a multi-room indoors experiment}

In order to test the robustness and the generalization capabilities of our architecture, we made several indoor navigation experiments in a $25 \times 15 \mathrm{~m}$ environment (our laboratory). For analysis purpose, we supervized the robot learning to recognize 19 places (each $1.5 \mathrm{~m}$ ) on a multi-room trajectory. The trajectory starts in one room, pass through a corridor and ends in a second room (mostly similar to the first one). Next, the robot followed 5 different parallel trajectories, imposed by a remote control. Visual recalibration is allowed (calibration driven by well recognized VPCs only, no periodic homing behavior).

Those five trajectories permit to cover a large space near the learned path, in order to test generalization capability (figure 4).

The visual recognition system allows great generalization capability (large place field) but present small perception mistakes due to cue redundancy. On contrary, the proprioceptive recognition system presents well-defined place fields without any ambiguity, but is subject to the classical cumulative error of odometry. This induces a very precise discrimination for small scales but a shifted localization for larger one. Results show interesting emergent characteristics since the merging mechanism keep a correct localization even if errors simultaneously happen on the 2 modalities. Merging modalities hightlights contingencies and reduces non-contingent activities. Two low-level activities at the same time are more coherent than a singural high-leveled one.

Finally, MPCs are robust to ambiguity and keep large generalization properties. To show the deterministic nature of the results, we repeated the experiment a 
A - Long range indoor experiment

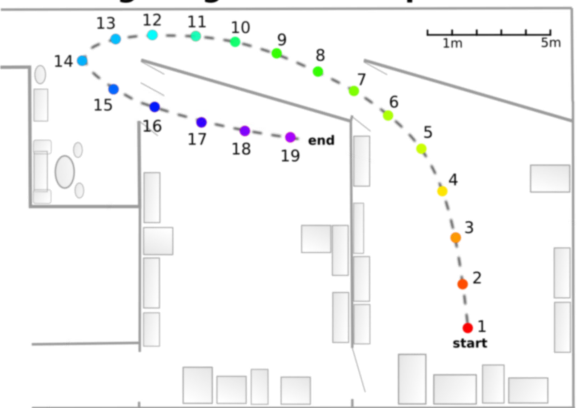

C - Grid recognition

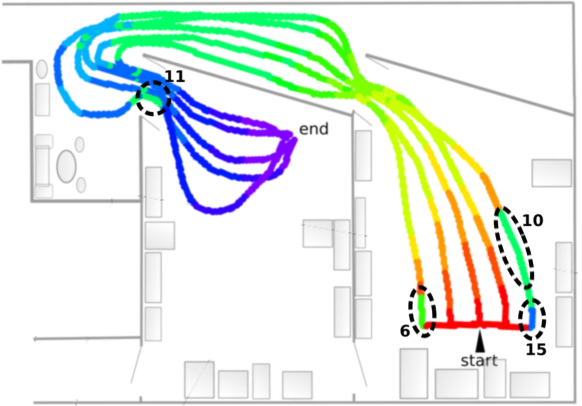

B - Visual recognition

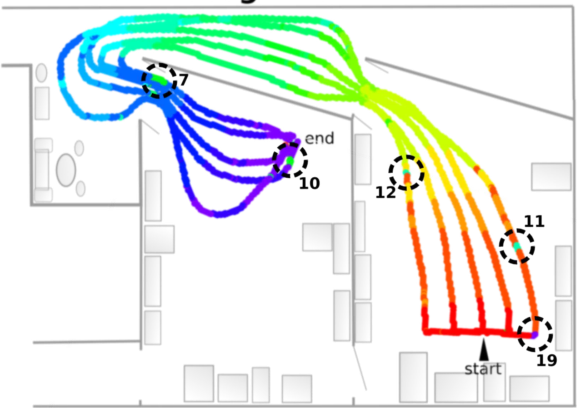

D - Multimodal recognition

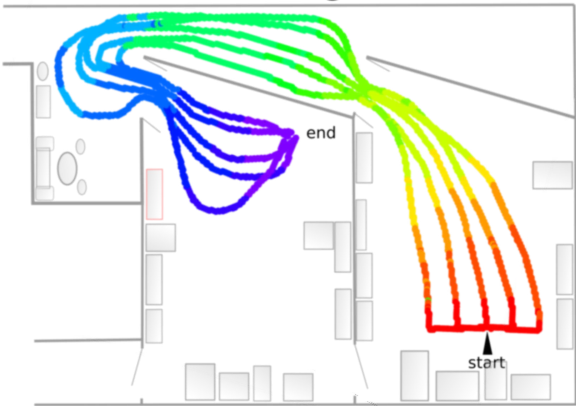

Fig. 4. Long range navigation in an indoor environment: A - Experimental setup: The robot learns 19 regularly-spaced places (each $1.5 \mathrm{~m}$ ), starting from a room, passing through a corridor and ending on a second room. B - Visual recognition obtained for 5 different trajectories. Each color is associated to one visual place cell. Results show great generalization capabilities but present ambiguities (dotted circles. Numbers correspond to perceived places.). C - Grid recognition for the same trajectories. Grid fields are smaller but without any ambiguities. A place is not recognized if the robot is too far away from the learned place. D - Multimodal recognition obtained by merging visual and grid place cells. The synergy of both modalities shows well defined areas even if the robot is far from the learned trajectory.

dozen of times in a changing environment (ambiant light and furnitures changing, persons moving). We also studied what happen when the robot is lifted, blindfolded, then transported to another place. If this place is already known and highly recognized, the robot recalibrates its path integration field to a previously learned value. We made several kidnapping events in order to test the robustness of this mechanism. Each times, the robot typically runs 5 meters before to recalibrate its odometry. Thanks to the merging mechanism, the perceived location never stays wrong for more than 2 meters. This recalibration mechanism allows the robot to always keep consistancy between vision and proprioception and never getting lost near learned locations.

\subsection{Topology matters : On the need for convolving grid cells}

In our model, all grids present binary fields (activated or not) so that the pattern generated by the conjunction of 3 grids is a three-steps stair shaped. Moreover the 3 differents modulo factors are relatively prime so that the activity of the 
conjunction pattern is often completely different of the previous one for only few movements. Narrow place cells are treated like there is no proximity distance between them. Each grid cell is considered as an orthogonal input so that grid networks doesn't benefit from any topology. That is the reason why we propose to convolve each grid network with a pyramidal-shaped mask, giving to the system more generalization capabilities. This technics allows to spread field activities over neighboring cells by using a natural torus topology [11], and so generates continuity. In return, it looses the ability to distinguish between 2 near places if the distance between them is smaller than the mask size (figure 5). We used this convolution method in the multi-room experiment presented in section 4.2. Without that convolution, the system can't work.
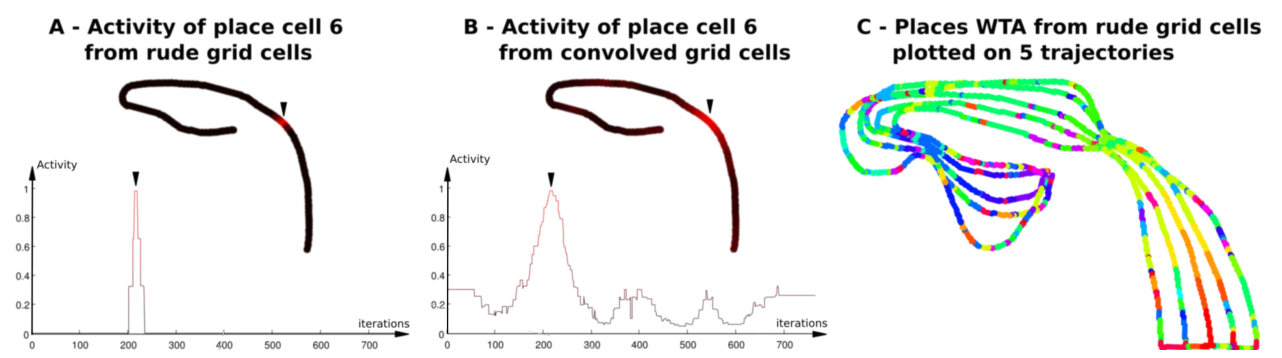

Fig. 5. Example of the diffusion mechanism used to generates topology. A - Activity of motor place cell 6 from binary grid cells, in space and time on a multi-room trajectory (before to be convolved). The place field is a thin three-steps stair shaped. B - Activity of motor place cell 6 from convolved grid cells. The convolution act as a diffusion mechanism spreading activity on neighboring grid cells. Motor place cells show gaussian shaped activity allowing generalization capabilities. C - Results obtained for winning motor place cells from binary grid cells (no diffusion) during the previous indoor experiment. It shows a lot of errors that can be easily removed by adding the diffusion mechanism.

Such method confront us with its biological plausibility since experiments made on rodent only show binary activities. It questions about how is it possible for mammals to correctly navigate using grid cells binary fields. We argue that the topology needed can naturally emerge from the large number of grid cells in rodent's brain.

\section{Discussion}

In this incremental design approach, one objective of our robotic experiments is to show the limitations of models (proof by failure). Hence, highligting the need to take into account new cerebral structures or interactions between them allows us to propose a more coherent model for a better understanding of explored brain structures.

Our experiments emphasized some issues while trying to scale our architectures to larger environment: ambiguities coming from the visual modality have been identified as the major problem. Consequently, we extended our architecture by modelling grid cells networks and we presented a robotic experiment that can 
account for their firing properties. Then, we present a simple merging mechanism exploiting these grid cells to desambiguate visual perception and generate robust multimodal place cells. We show that it succesfully overcome the perception ambiguity problem and it stay robust even if the system is blindfolded or kidnapped, then lifted to another place. Moreover, results show an emergent characteristic hightlighting contingency and reducing singular activities since the merging mechanism keeps a coherent localization even if errors simultaneously happen on both modalities.

We also underline the need to spread grid cells binary activity to neighboring cells to create a topology inducing interesting generalization properties. It questions about the biological plausibility of such ad-hoc method. We claim that such topology can naturally emerge from the large number of grid cells in rodent's brain.

Our current work focuses on switching navigation strategies according to an emotional metacontroller based on bayesian inferences. In the same time, we are performing long range (several kilometers) outdoor navigation experiments based on these models.

\section{Acknowledgment}

We would like to thank AUTO-EVAL and the NEUROBOT French ANR project.

\section{References}

1. J. OKeefe and J. Dostrovski. The hippocampus as a spatial map. preliminary evidence from unit activity in the freely-moving rat. Brain Research, 34:171175, 1971.

2. N. Cuperlier, M. Quoy, and Ph. Gaussier. Neurobiologically inspired mobile robot navigation and planning. Frontiers in NeuroRobotics, 1(1), 2007.

3. E. J. Markus, C. A. Barnes, B. L. McNaughton, V. L. Gladden and W. E. Skaggs. Spatial information content and reliability of hippocampal CA1 neurons: Effects of visual input. (2004) 10.1002/hipo.450040404.

4. RU Muller, JL Kubie and JB Ranck Jr. Spatial firing patterns of hippocampal complex-spike cells in a fixed environment.(1987) The Journal of Neuroscience, 1 July 1987, 7(7): 1935-1950;

5. GJ Quirk, RU Muller and JL Kubie. The firing of hippocampal place cells in the dark depends on the rat's recent experience. The Journal of Neuroscience, 1 June 1990, 10(6): 2008-2017;

6. F. Schenk, M. C. Grobety, P. Lavenex and H.-P. Lipp. Dissociation between Basic Components of Spatial Memory in Rats. Science Series D: 1995, Volume 82, Part III, 277-300.

7. F. Schenk and P. Lavenex. Olfactory traces and spatial learning in rats. (1998) Institut of Physiology, University of Lausanne.

8. D. G. Wallace, D. J. Hines, S. M. Pellis and I. Q. Whishaw. Vestibular Information Is Required for Dead Reckoning in the Rat. The Journal of Neuroscience, 15 November 2002, 22(22): 10009-10017;

9. Hafting T, Fyhn M, Molden S, Moser MB, Moser EI. Microstructure of a spatial map in the entorhinal cortex. Nature. 2005 Aug 11; 436(7052): 801-6. 
10. M. C. Fuhs and D. S. Touretzky. A Spin Glass Model of Path Integration in Rat Medial Entorhinal Cortex. (2006) 26(16): 4266-4276;

11. B. L. McNaughton, F. P. Battaglia, O. Jensen, E. I Moser M-B. Moser. Path integration and the neural basis of the 'cognitive map'. Nature Reviews Neuroscience 7, 663-678 (August 2006).

12. N. Burgess, C. Barry and J. O'Keefe. An oscillatory interference model of grid cell firing. (2007) 10.1002/hipo.20327.

13. I. R. Fiete, Y. Burak and T. Brookings. What Grid Cells Convey about Rat Location. The Journal of Neuroscience, 2 July 2008, 28(27): 6858-6871;

14. P. Gaussier, J. P. Banquet, F. Sargolini, C. Giovannengeli, E. Save and B. Poucet. A model of grid cells involving extra hippocampal path integration, and the hippocampal loop. Journal of Integrative Neuroscience, Vol. 6, No. 3 (2007) 447476.

15. J.OKeefe and N. Nadel. The hippocampus as a cognitive map. Clarendon Press, Oxford, 1978.

16. P. Gaussier, A. Revel, J.-P. Banquet, and V. Babeau. From view cells and place cells to cognitive map learning: processing stages of the hippocampal system. Biological Cybernetics, 86:1528, 2002.

17. J.-P. Banquet, P. Gaussier, M. Quoy, A. Revel, and Y. Burnod. A hierarchy of associations in hippocampo-cortical systems: Cognitive maps and navigation strategies. Neural Computation, 17(6):13391384, June 2005.

18. C. Giovannangeli, P. Gaussier, and J. P. Banquet. Robustness of visual place cells in dynamic indoor and outdoor environment. International Journal of Advanced Robotic Systems, 3(2):115124, June 2006.

19. B. Kolb and R. Tees. The Cerebral Cortex of the Rat. MIT Press, 1990.

20. W. A. Suzuki, E. K. Miller, and R. Desimone. Object and place memory in the macaque entorhinal cortex. J Neurophysiol, 78(2):10621081, Aug 1997.

21. R. D. Burwell and D. M. Hafeman. Positional ring properties of postrhinal cortex neurons. Neuroscience, 119(2):577588, 2003.

22. P. Gaussier and S. Zrehen. Perac: A neural architecture to control artificial animals. Robotics and Autonomous Systems, 16(2-4):291 320, 1995. Moving the Frontiers between Robotics and Biology.

23. A. Pouget, S. Deneve J-R. Duhamel. A computational perspective on the neural basis of multisensory spatial representations. Nature Reviews Neuroscience 3, 741747 (1 September 2002) — doi:10.1038/nrn914.

24. B. Widrow and M.E. Hoff, Jr. Adaptive Switching Circuits. IRE WESCON Convention Record, 4:96-104, August 1960.

25. Simon Haykin. Adaptive Filter Theory. Prentice Hall, 2002, ISBN 0-13-048434-2

26. Pavlov, I. P. (1927). Conditioned reflexes. London: Routledge and Kegan Paul.

27. T. Strosslin, D. Sheynikhovich, R. Chavarriaga, and W. Gerstner (2005). Robust self-localisation and navigation based on hippocampal place cells. NEURAL NETWORKS 18 (9): 1125-1140.

28. Arleo, A., Smeraldi, F., Hug, S., Gerstner, W. (2001). (T. K. Leen, T. G. Dietterich, V. Tresp, Eds.) Advances in neural information processing systems, 1(ii), 89-95. Citeseer.

29. J. M. Herrmann, K. Pawelzik, T. Geisel (1999) Self-localization of autonomous robots by hidden representations. Autonomous Robots 7:1, 31-40.

30. M. J Mataric, A Distributed Model for Mobile Robot Environment-Learning and Navigation, MIT EECS Master's Thesis, Jan 1990. 